\title{
GW23-e2123 QUANTITATIVE STUDY OF LUMBAR SPINE BONE MINERAL DENSITY AND CORONARY ARTERY CALCIFICATION IN ASYMPTOMATIC POSTMENOPAUSAL WOMEN
}

doi:10.1136/heartjnl-2012-302920a.182

Zhao Yuan, Xing Yan, Liu Wen-ya, Yang Wen, Wang Hai-tao, Liu Wen-ya. First Affiliated Hospital, Xinjiang Medical University

Objectives To analyse the result of lumbar spine bone mineral density and coronary artery calcification, and to investigate the correlation between the change of bone mineral density and coronary atherosclerosis in asymptomatic postmenopausal women and their probable mechanism.

Methods 112 patients underwent quantitative CT (QCT) of lumbar vertebrae for bone mineral density (BMD) and multi-slice spiral CT (MSCT) of coronary artery for calcification score and totals calcification score (TCS), filled out interview sheet for the details of general condition, lifestyle and other factors. All patients were divided into control osteopenia and osteoporosis groups based on the $\mathrm{T}$ score of the lumbar spine. The clinical data and $\mathrm{BMD}$ were compared among three groups by using an analysis of variance (ANOVA) for continuous variable. Analysis of osteoporosis and factors was done by using multiple regression.

Results age was significantly older in group of osteoporosis than other two groups $p<0.05$ ). Years of menopause were significantly different between the group of osteoporosis and the group of control, BMD of lumbar spine and TCS were significantly different among three groups. P HDL were lower in group of osteoporosis than other two groups, but there was no significant difference ( $p>0.05$ ). Multiple regression analysis showed that the factors for TCS in patients included age smoking high density lipoprotein $(\mathrm{HDL})$ and $\mathrm{BMD}$, while age $\mathrm{TC}$ were risk factors, $(\mathrm{HDL})$ and $\mathrm{BMD}$ were protect factors, There was negative correlation between the coronary artery calcification score and BMD.

Conclusions This study provides initial data suggesting that postmenopausal women who with osteopenia or osteoporosis may have a higher risk of developing coronary atherosclerosis, Osteoporosis was correlated with coronary atherosclerosis and $\mathrm{BMD}$ could be considered as an new index for assessment coronary atherosclerosis. 